\title{
Eczema herpeticum in early pregnancy
}

\author{
Eunjung L. Kim MD, Benjamin Hohmuth MD MPH
}

Cite as: CMAJ 2017 April 3;189:E505. doi: 10.1503/cmaj.151544
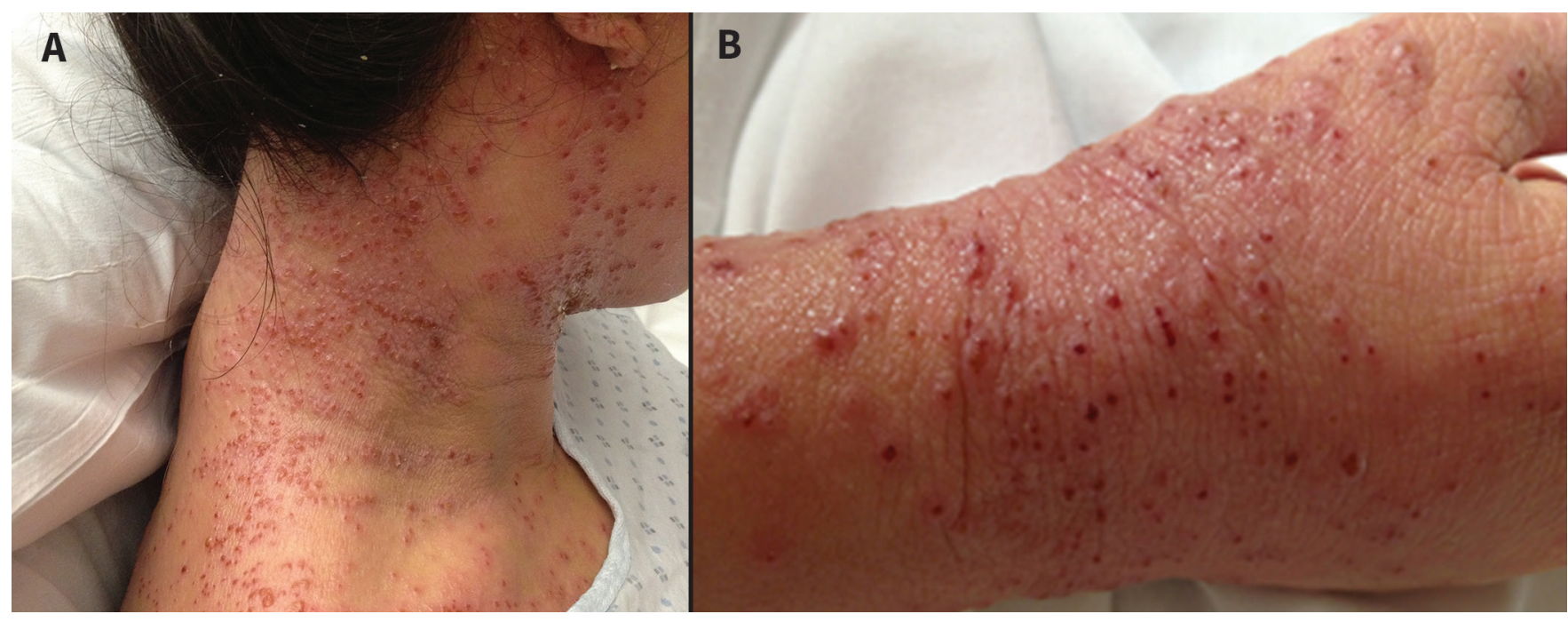

Figure 1: Skin rash, consisting of crusted plaques and vesicles, on the face and neck (A) and the right hand (B) of a 25-year-old woman at 12 weeks gestation with eczema herpeticum.

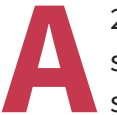

25 year-old woman with a history of atopic dermatitis presented to the emergency department with a generalized skin rash. She was at 12 weeks gestation in her first pregnancy. The skin lesions first appeared on her chest in an area where she had symptomatic eczema. These lesions were painful and diffuse, and were located on her face (Figure 1A), trunk and extremities (Figure 1B). The lesions consisted of crusted plaques and vesicles that drained clear fluid. Her body temperature was $101.1^{\circ} \mathrm{F}$ $\left(38.4^{\circ} \mathrm{C}\right)$, her heart rate was 137 beats/min, and her leukocyte count was $11.8 \mathrm{~K} / \mu \mathrm{L}\left(11.8 \times 10^{9} / \mathrm{L}\right)$.

Given her history of atopic dermatitis and diffuse vesicular lesions, we considered eczema herpeticum in the initial differential diagnosis and started acyclovir intravenously. Pelvic ultrasonography confirmed a 12-week-old viable intrauterine pregnancy. A Tzanck test from a skin scraping showed multinucleated giant epithelial cells. Levels of antibodies for herpes simplex virus (HSV) immunoglobulins M and G were elevated for HSV type 1.

By day 4, the patient had been afebrile for 48 hours and her skin eruptions had improved. We changed treatment with acyclovir from intravenous to oral (200 $\mathrm{mg}$ five times daily) for a total of 10 days of treatment, and the patient was discharged home.

Eczema herpeticum is a diffuse skin infection caused by HSV-1/2. It generally occurs in individuals with chronic skin disorders, such as atopic dermatitis. It can be spread by direct contact of susceptible skin with HSV or reactivated from previous infection. Immunosuppression and pregnancy may predispose patients to severe visceral dissemina- tion of HSV that can spread rapidly and is associated with high mortality. ${ }^{1}$ Delayed diagnosis and treatment in women who are pregnant can lead to fulminant infection, which further increases risk of maternal death, preterm labour, neonatal HSV infection or fetal death.

Acyclovir, valacyclovir and famciclovir have been used safely in the first trimester of pregnancy for infections caused by HSV. ${ }^{2}$ There are no controlled studies or current guidelines on managing this infection with antiviral agents in pregnant women; however, it appears safe to transition from intravenous to oral therapy when the skin rash improves and the patient has been afebrile for at least 48 hours.

\section{References}

1. Schiffer JT, Corey L. Herpes simplex virus. Mandell, Douglas, and Bennette's Principles and Practice of Infectious Disease. 8th ed. Philadelphia: Elsevier Saunders; 2014:1721,1729.

2. Pasternak B, Hviid A. Use of acyclovir, valacyclovir, and famciclovir in the first trimester of pregnancy and the risk of birth defects. JAMA 2010;304:859-66.

\section{Competing interests: None declared.}

This article has been peer reviewed.

The authors have obtained patient consent.

Affiliations: Department of Internal Medicine (Kim), Englewood Hospital and Medical Center, Englewood, NJ; Department of Hospital Medicine (Hohmuth), Geisinger Medical Center, Danville, Penn.

Correspondence to: Eunjung Liliana Kim, maylilith@gmail.com 